\title{
Potential of SPR Sensors Based on Multilayer Interfaces With Gold and LHM for Biosensing Applications
}

\author{
Abdellatif CHERIFI* and Benamar BOUHAFS \\ University of Tlemcen, Theoretical Physics Laboratory, Physics of Department, Tlemcen, 13000, Algeria \\ ${ }^{*}$ Corresponding author: Abdellatif CHERIFI \\ E-mail: Cherifi_physics@yahoo.com
}

\begin{abstract}
Recently, the subject on "plasmonics" has received significant attention in designing surface plasmon resonance (SPR) sensors. In order to achieve extremely high-sensitivity sensing, multilayered configurations based on a variety of active materials and dielectrics have been exploited. In this work, a novel SPR sensor is proposed and investigated theoretically. The structure, analyzed in attenuated total reflection (ATR), consists of multilayer interfaces between gold and a metamaterial (LHM) separated by an analyte layer as a sensing medium. By interchanging between gold and LHM, under the effect of the refractive index (RI) of analyte set to be in the range of 1.00 to 1.99, the sharp peak reflectivity at the SPR angle takes two opposite behaviors predicted from the transfer matrix method. At the threshold value of 1.568 of the refractive index of analyte and when the LHM is the outer medium, the layered structure exhibits a giant sharp peak located at $43^{\circ}$ of intensity up to $10^{5}$ due to the Goos-Hànchen effect. With respect to the refractive index (RI) change and thickness of analyte, the characteristics (intensity, resonance condition, and quality factor) of the SPR mode, which make the proposed device have the potential for biosensing applications, have been analytically modelized.
\end{abstract}

Keywords: Plasmonic; goos-Hànchen $(\mathrm{GH})$ effect; SPR sensor systems; sub-wavelength scale

Citation: Abdellatif CHERIFI and Benamar BOUHAFS, "Potential of SPR Sensors Based on Multilayer Interfaces With Gold and LHM for Biosensing Applications," Photonic Sensors, 2017, 7(3): 199-205.

\section{Introduction}

The surface plasmon resonance (SPR) phenomenon known for a long time has been used in large practical applications such as terahertz $(\mathrm{THz})$ filter [1], plasmon enhanced solar cell [2, 3], plasmonic detectors [4], optical antenna [5], biological analysis [6], and chemical sensors [7]. This resonant phenomenon corresponds to a coupling process between a monochromatic light and conduction electrons on the boundary of a metal surface and a dielectric. With an attenuated total reflection method (ATR), the SPR mode is strongly sensitive to both thickness and refractive index of the dielectric. Due to this characteristic, SPR becomes a highly efficient mechanism in designing plasmonic sensor systems. In 1982, the first application of SPR was done by Liedberg et al. [8, 9]. However, the efficient way of manipulating the characteristics of the electromagnetic field correlated to SP in a nanoscale, remains related particularly to the involved interface properties. Therefore, a multilayer SPR sensor leads to achieving other limits that can be preferable to those 
obtained according to the conventional Kretschmann configuration [10]. In the above configuration, when a p-polarized light is incident on the prism-metal interface and the reflectance is measured versus the incident angle, a sharp dip occurs at the resonance angle beyond the critical angle. Within the medium to be sensed in contact with the metal layer [11], the SP field evolutes in an evanescent character into both mediums of the interface. The resolution of an SPR-sensor in terms of line width and dip position of SPR-reflectivity depends on the environmental index change $[12,13]$. As an example of SPR-sensor, we cite the optical sensing based on chalcogenide prism and graphene layers in addition to the conventionally used Au-layer for the detection of bimolecular analyte [14], periodic nanostructures $\mathrm{Au}$ nano-gratings [15], etc.

In recent years, plasmonic metamaterials hold a new promise for biosensing with unprecedented sensitivity and specificity. Metamaterials are composite materials whose permittivity $\varepsilon$ and permeability $\mu$ are both negative. Thus, metamaterials are described by the negative refraction index $n=-(\mu \varepsilon)^{1 / 2}$. In this topic, the first discussion on metamaterials type was advanced in 1968 by Veselago [16]. In 1999, Pendry et al. identified a practical way to construct left-handed metamaterials (LHMs) of a different behavior which did not follow the conventional right hand rule [17].

Recently, researchers have successfully put forward many different biosensor configurations with greatly enhanced sensitivity to the LHM variation. For example, Schueler et al. [18] reviewed the LHM inspired composite transmission line microwave sensors. Chen et al. [19] reviewed the LHM application in sensing with an emphasis on split ring resonator-based sensors. Yang et al. studied LHM sensors based on a single metamaterials particle with the advantages of an easy fabrication and experimental robustness [20]. Upadhyar et al. [21] used LHM instead of metals to investigate SPs in low-wavelength sensors [22], and Zheludev [23] analyzed the future development of
LHMs and pointed out that the sensing application represents a growing area.

Very recently, Pal et al. [24] proposed SPR sensors based on bi-LHMs with gold as an inner core and compared the above structure to another with two dielectric media instead of LHM. On the analysis of the reflectivity profile, the authors [24] pointed out the advantage of using the metamaterials to further enhance SPR sensor sensitivity. Some researchers have demonstrated typical multilayer devices as biosensors that provide sensitivity almost 6-fold higher than that of the conventional SPR sensor [25]. Sensitivity enhancement of the SPR-sensorwas the discussion subject in [26], in which a bi-metallic structure was established and a comparative analysis with the conventional SPR configuration to that of an SPR sensor of four and five layered LHM media was made.

The main objective of this work focuses on exploring SPR on a tunable nanocavity where an analyte (sensing medium) is embedded between symmetric interfaces gold/LHM. To interpret the SPR response, the transfer matrix method, applied as a repeated Fresnel's equations, conducts to express the reflection coefficient within the designed structure. The above response is calculated in the angular mode at 738-nm wavelength. By optimizing the thicknesses of the media to get a minimum dip, we investigate particularly the effect of the refractive index $n_{s}$ on the SPR profile. The later spectrum leads to evaluating angular-sensitivity defined as the ratio between the SPR angle shift and refractive index, $n_{s}$ and $Q$-factor. Finally, the best parameter conditions can be concluded to identify the best $Q$-factor and sensitivity.

\section{Theory and characteristics of the considered sensor}

As shown in Fig. 1, we consider a multilayer wave guide geometry with $N$ layers. Each medium is subscribed by of a thickness $d_{k}$, permittivity $\varepsilon_{k}$, and permeability $\mu_{k}$. This multilayer is disposed onto the base of a prism coupler. In the whole work, two arrangements have been analyzed, one is 
Au-analyte- LHM, and the other is LHM-analyte-Au for which the respective reflectivity is deduced by means of the transfer matrix method.

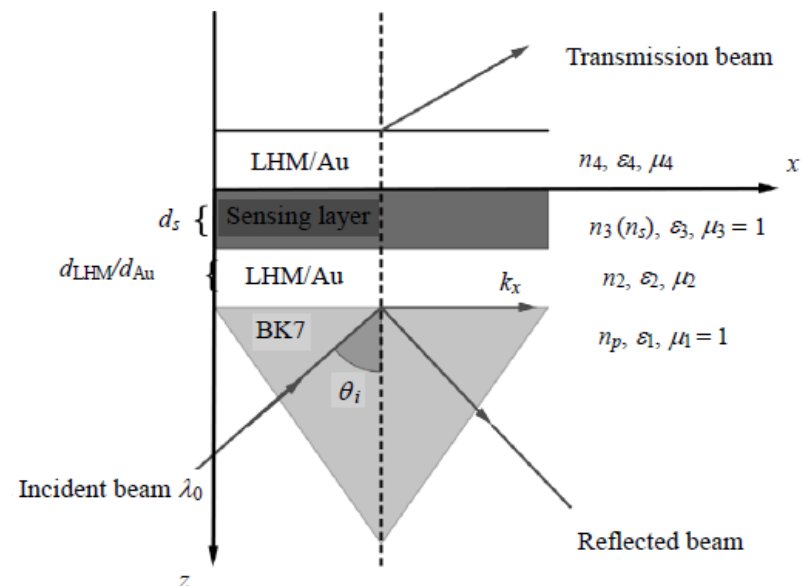

Fig. 1 Schematic illustration of the composite waveguide sensor under study.

In this method, the tangential fields at the first boundary $z_{1}$ are related to those at the last boundary $z_{n}$ as it will be explained below. So, when the structure is irradiated with a light beam of transverse magnetic (TM or $\mathrm{p}$ ) polarization under the incidence angle $\theta_{i}$ through a BK7-glass prism, consequently a field distribution occurs in the four media. The relationships between electromagnetic fields $\left(\mathbf{E}_{1}, \mathbf{B}_{1}\right)$, $\left(\mathbf{E}_{2}, \mathbf{B}_{2}\right),\left(\mathbf{E}_{3}, \mathbf{B}_{3}\right)$, and $\left(\mathbf{E}_{4}, \mathbf{B}_{4}\right)$ in the four media obtained by using phase change due to light passing through the different layers denoted as $k$ take the forms as

$$
\left\{\begin{array}{l}
E_{k}=\left(\mathrm{e}^{-\mathrm{i} \beta_{k}} \cos \beta_{k}\right) E_{k+1}-\left(\mathrm{i} \frac{\mathrm{e}^{-\mathrm{i} \beta_{k}}}{q_{k}} \sin \beta_{k}\right) B_{k+1} \\
B_{k}=\left(\mathrm{i} q_{k} \mathrm{e}^{-\mathrm{i} \beta_{k}} \sin \beta_{k}\right) E_{k+1}-\left(\mathrm{e}^{-\mathrm{i} \beta_{k}} \cos \beta_{k}\right) B_{k+1}
\end{array}\right.
$$

where $\beta_{k}$ is the optically admittance, and $q_{k}$ is the phase factor. For each medium subscribed by $k$, these quantities are given as follows:

$$
q_{k}=\left(\mu_{k} / \varepsilon_{k}\right)^{1 / 2} \cos \theta_{k}=\frac{\left(\varepsilon_{k} \mu_{k}-n_{1}^{2} \sin ^{2} \theta_{1}\right)^{1 / 2}}{s_{k}}
$$

and

$$
\beta_{k}=\frac{2 \pi d_{k}}{\lambda}\left(\varepsilon_{k} \mu_{k}-n_{1}^{2} \sin ^{2} \theta_{1}\right)^{1 / 2} .
$$

For the proposed SPR sensor of four layers, the characteristics matrix is given as

$$
\left[\begin{array}{l}
\mathbf{E}_{1} \\
\mathbf{B}_{1}
\end{array}\right]=\mathbf{M}\left[\begin{array}{l}
\mathbf{E}_{4} \\
\mathbf{B}_{4}
\end{array}\right]
$$

where the matrix $\mathbf{M}$ takes the form as follows:

$$
\begin{aligned}
\mathbf{M}= & \prod_{k=2}^{4}\left[\begin{array}{cc}
\cos \beta_{k} & -\mathrm{i} \sin \beta_{k} / q_{k} \\
-\mathrm{i} q_{k} \sin \beta_{k} & \cos \beta_{k}
\end{array}\right]= \\
& {\left[\begin{array}{ll}
M_{11} & M_{12} \\
M_{21} & M_{22}
\end{array}\right] . }
\end{aligned}
$$

Finally, the amplitude of the reflection coefficient for p-polarized incident wave is given as [27]

$$
r_{p}=\left|\frac{\left(M_{11}+M_{12} q_{s}\right) q_{1}-\left(M_{21}+M_{22} q_{s}\right)}{\left(M_{11}+M_{12} q_{s}\right) q_{1}+\left(M_{21}+M_{22} q_{s}\right)}\right|^{2}
$$

and the reflectivity for the p-polarized light can be analyzed with respect to the angle of incidence $\theta_{i}$ as follows:

$$
R_{P}=\left|r_{P}\right|^{2} .
$$

Using (7), the resolution of the SPR peak that exhibits the structure can be determined by adjusting thicknesses, $d_{k}$ and refractive index, $\varepsilon_{k}$ of the mediums (Cf. Fig. 1 for notations). From this SPR response, the determination of the resonance condition where the reflectivity, $R_{p}\left(\theta_{i}=\theta_{\mathrm{SPR}}\right)$ drops to a minimum value, provides the propagation wavelength, $\lambda_{\mathrm{SPR}}$ of the surface plasmon as:

$$
\lambda_{\mathrm{SPR}}=\frac{\lambda_{0} / n_{p}}{\sin \theta_{\mathrm{SPR}}}
$$

where $\lambda_{0}$ is the free space wavelength in vacuum.

Generally, the performance of plasmonic sensors is evaluated in terms of angular sensitivity, resolution or detection limit (DL), and quality factor $Q$. Based on the reflectivity's profile, with a fixed wavelength $\lambda_{0}$, the angular sensitivity is the ratio between the changes in SPR angle $\partial \theta_{\mathrm{SPR}}$ and the change $\partial n_{s}$ [12] expressed as follows:

$$
S=\frac{\partial \theta_{\mathrm{SPR}}}{\partial n_{s}} \text { or } \frac{\partial \lambda_{\mathrm{SPR}}}{\lambda n_{s}} .
$$

For the quality factor, $Q$ that can be evaluated from the linewidth at $50 \%$ of the SPR peak [28] noted as $\left(\Delta \theta_{0.5}\right)^{-1}$, which is given as follows:

$$
Q=\left(\frac{\Delta \theta_{0.5}}{\tan \theta_{\mathrm{SPR}}}\right)^{-1} \text { or }\left(\frac{\Delta \lambda_{0.5}}{\tan \lambda_{\mathrm{SPR}}}\right)^{-1} \text {. }
$$

\section{Results and discussions}

In order to outline the resolution of the proposed 
SPR sensor as illustrated in Fig. 1, the reflectivity is calculated and plotted numerically for two arrangements between $\mathrm{Au}$ and LHM. Thus, three main parameters, namely intensity, detection accuracy, and quality factor, are evaluated particularly under the influence of the refractive index $n_{s}$ of analyte. The refractive index of BK7-prism is set to be 1.52 [29].

Using (7), the angular profiles of SPR response are displayed in Figs. 2, 3, 4, and 5. It is noted that the specific arrangements that correspond to these data are reported in the insert of the figures. In these plots, we have taken for gold the complex permittivity expressed from Drude-Lorentz model, $\varepsilon_{\mathrm{Au}}=-21.3+1.34 \mathrm{i}$ as tabulated in [30]. The electric permittivity and magnetic permeability for the LHM, are -33.5 and -11 , respectively which are listed in [31]. The sensing medium termed analyte of thickness, $d_{s}=385 \mathrm{~nm}$, has the refractive index (RI) $n_{s}$, which varies from 1.00 to 1.66 .

In the above conditions, under the effect of $n_{s}$ of the sensing medium, the SPR curves are simulated in Fig. 2. These data indicate that an increase in RI contributes firstly to the trap single SPR mode or double SPR mode and secondly to reduce the linewidth, i.e. high quality factor.

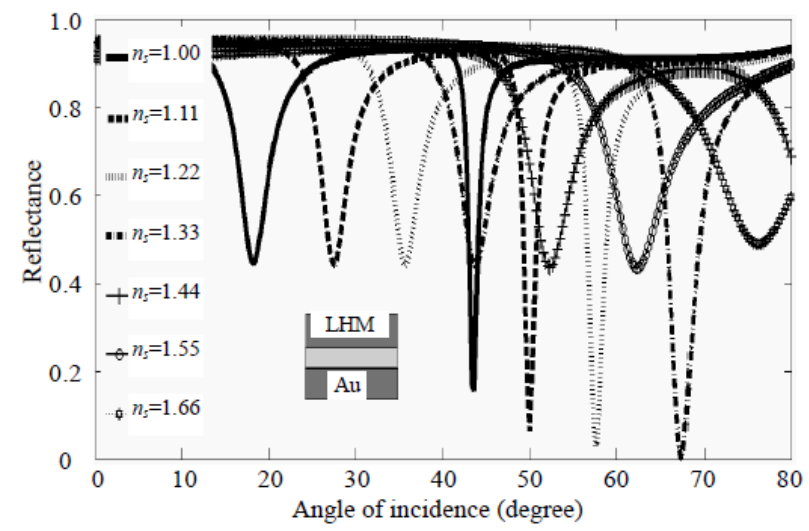

Fig. 2 Simulated SPR curves with different refractive indexes of sensing layer (thickness $385 \mathrm{~nm}$ ) stacked between $\mathrm{Au}$ the layer (refractive index $\varepsilon_{\mathrm{Au}}=-21.3+1.34 \mathrm{i}$ thickness $40 \mathrm{~nm}$ ) and left-handed materials (LHM) of $\left\{\varepsilon_{\mathrm{LHM}}, \mu_{\mathrm{LHM}}\right\}=\{-33.5$, $-11\}$.

According to Fig. 3, it is seen that an increase in $n_{s}$ from 1.33 to 1.35 leads to the best enhancement effect determined on the SPR-mode termed SP1 since we have already observed the zero-reflectance in a narrower angular range of $68^{\circ}-70^{\circ}$.

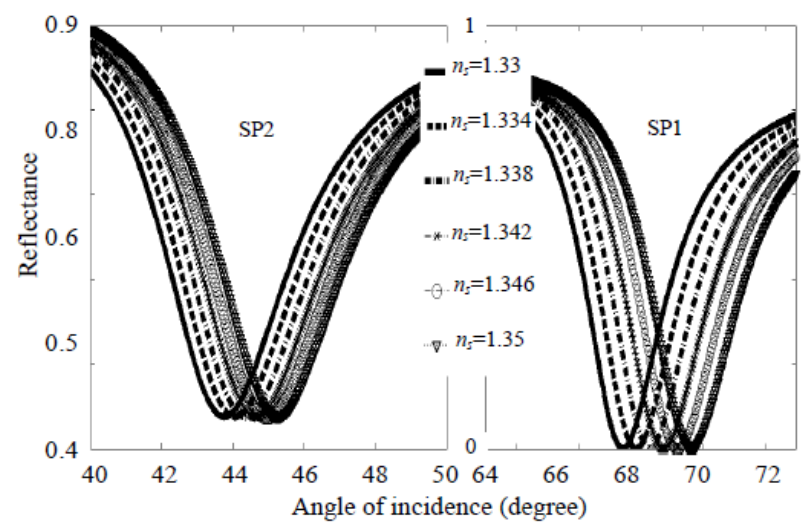

Fig. 3 Calculated p-reflectance with six different refractive indexes, $n_{s}$ of the sensing layer. The other parameters are the same as in Fig. 2.

It is not the case of the SPR-mode termed SP2 which is excited at the lower angle than SP1. In other hand, when tuning the $n_{s}$, an apparent discrepancy in term of the resolution is seen on the minimum dips of SP1 and SP2.

The minimum of the intensity remains constant, i.e. $R_{\mathrm{SPR}}=0.44$ of the SP2 mode and where the characteristics of SP1 are $\theta_{\mathrm{SPR}}=67.42^{\circ}, R_{\mathrm{SPR}}=3 \times$ $10^{-5}$, D.A. $=0.313 /{ }^{\circ}, \mathrm{FWHM}=3.19^{\circ}$, and $Q=43.23$ at $n_{s}=1.33$. In addition, the best parameters of SPR1 are $\theta_{\mathrm{SPR}}=27^{\circ}$, D.A. $=11.11 /{ }^{\circ}$, and $Q=325$ which in turn measure the resonance angle with optimizing both $\mathrm{Au}$ and sensing layer thicknesses $70 \mathrm{~nm}$ and $240 \mathrm{~nm}$, respectively. Guo et al. [28] obtained the angular sensitivity increasing as $103 \%$ RIU in a short range mode method, $97 \%$ RIU in a typical single mode method, and $70 \%$ RIU in a metallic method [32]. Additionally, the angular sensitivity of the SPR sensor based on prism was reported increasing from $94.66^{\circ} / \mathrm{RIU}$ to $204.41 \%$ RIU, but it went with an increase in FWHM from $2.24^{\circ}$ to $4.36^{\circ}$ [33].

A similar study of the reflectance is simulated for another arrangement between gold and LHM, which is depicted in Fig.4. The data show that with an active LHM of 720-nm thickness, the reflectance takes an opposite phase compared with the first arrangement $\mathrm{Au} /$ analyte/LHM structure. An increase in RI, $n_{\mathrm{s}}$ in the range of 1.00 to 1.99 causes an 
optical amplification of the SPR peak which is launched in a large angular range from $20^{\circ}$ to $80^{\circ}$. Here we specify that an increase inthe RI, $n_{s}$ in the restricted range of 1.44 to 1.66 gives rise to the Goos-Hànchen effect observed on the single SPR mode whose full width at half maximum is the narrowest. This structure exhibits one single SPR mode whose maximal intensity can be manipulated on the change in RI of the sensing from 1.00 to 1.55. The Goos-Hànchen peak shift has the best parameters as $\theta_{\mathrm{SPR}}=41.85^{\circ}, R_{\mathrm{SPR}}=3939$, D.A. $=$ $100 /{ }^{\circ}\left(\mathrm{FWHM}=0.01^{\circ}\right)$, and $Q=5134$ at $n_{s}=1.55$. For the particular RI, $n_{s}=1.22$, on the p-reflectance profile the structure exhibits two SPR modes with a large discrepancy between their respective intensities. The first dips denote SP1 (see left) with the parameters $\theta_{\mathrm{SPR}}=51.16^{\circ}, R_{\mathrm{SPR}}=0.0745$, D.A. $=$ $8.33 /^{\circ}\left(\mathrm{FWHM}=0.12^{\circ}\right)$, and $Q=593.1$, and the second denote SP2 (see right) with $\theta_{\mathrm{SPR}}=18.55^{\circ}$, $R_{\mathrm{SPR}}=0.34$, D.A. $=7.14 /^{\circ}\left(\mathrm{FWHM}=0.14^{\circ}\right)$, and $Q=$ 137.4. The inset shows the maximum resolution (detection limit) is D.A.max $=125 /{ }^{\circ}(\mathrm{FWHM}=$ $0.008^{\circ}$ ).

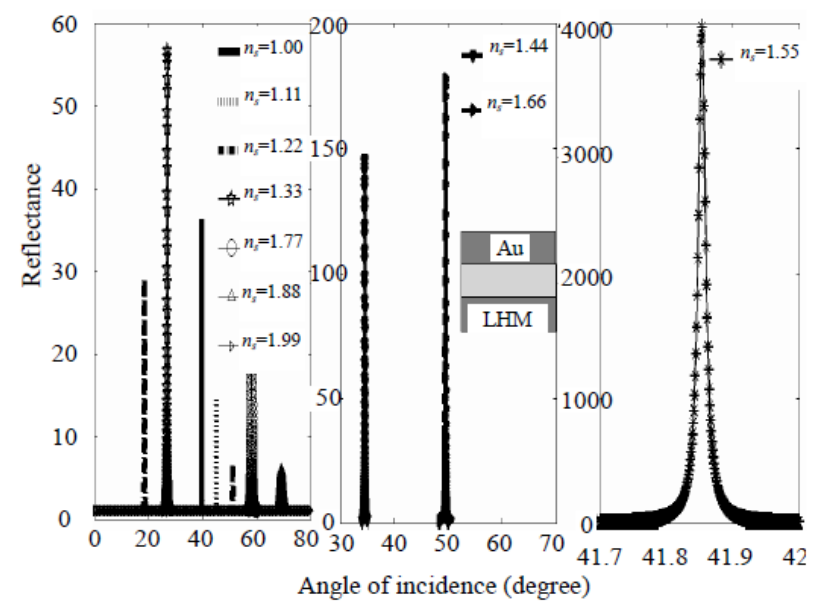

Fig. 4 Simulated SPR curves with different refractive indexes of the sensing layer (thickness $360 \mathrm{~nm}$ ) stacked between left-handed materials (LHM), (refractive index $\left\{\varepsilon_{\text {LHM }}\right.$, $\left.\mu_{\mathrm{LHM}}\right\}=\{-33.5,-11\}$, thickness $720 \mathrm{~nm}$ ) and the Au layer (refractive index $\left.\varepsilon_{\mathrm{Au}}=-21.3+1.34 \mathrm{i}\right)$.

From Fig. 5, the reflection efficiency of the peak increases slightly with an increase in the refractive index of the sensing layer from 1.33 to 1.55. Additionally, we can see that $n_{s}$ induces the shift of
SPR from $27.04^{\circ}$ towards a higher angle of the incident angle. One can also observe a particular influence on the reflectance intensity, as $R\left(\theta_{\mathrm{SPR}}=\right.$ $\left.43.07^{\circ}\right)=1.18 \times 10^{5}$ for $n_{s}=1.568$, and a high quality factor is estimated to be 6000 . The last issue that must be addressed is the question of the drop in the reflectance peaks for $\theta_{\mathrm{SPR}} \geq 43.04^{\circ}$ for $n_{s}>1.552$ of the sensing layer. This is simply due to the cut-off function of the RI associated to a nonabsorbent sensing layer. Finally, in the conditions of the parameters specified in left Fig. 5, a linear fit is predicted for SPR in RI change 1.33 to 1.42 , i.e. the parameters linearity with equation as $\theta_{\mathrm{SPR}}=70.136 \times$ $n_{s}-66.244\left(^{\circ}\right)$. And $Q=1000 \times n_{s}-1005$.

With error $\pm 0.009, \pm 7.54 \times 10^{-13}$ respectively and quadratic equation as $R_{\mathrm{SPR}}=3330.4 \times n_{s}{ }^{2}-8548 \times$ $n_{s}+5535.4$ at $n_{s}>1.43$ (right Fig. 5), the linearity equation of the resonance angle is $\theta_{\mathrm{SPR}}=67.72 \times n_{s}$ 63.13 with error \pm 0.0095 , and other parameters of SPR are nonlinearity.

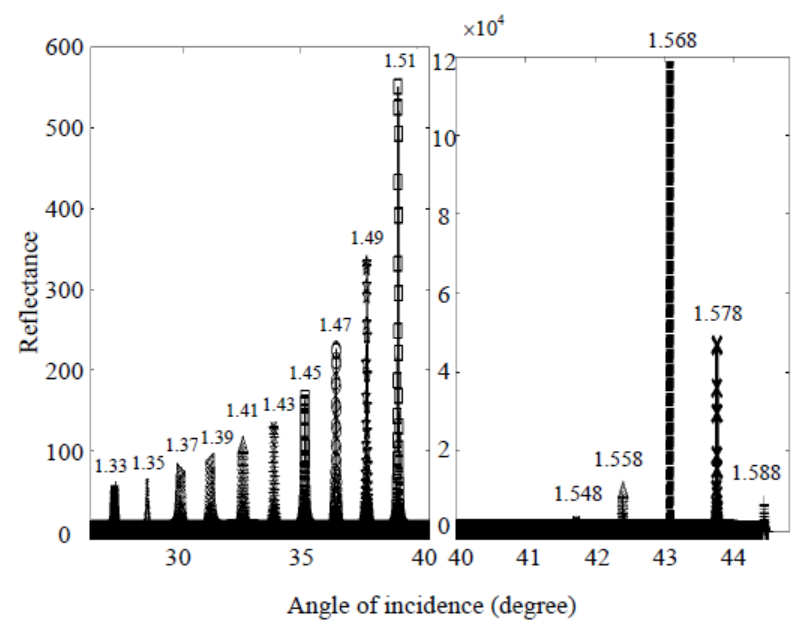

Fig. 5 Calculated reflectance for different refractive indexes of the sensing layer with different refractive indexes of the sensing layer, $n_{s}=1.33,1.35,1.37,1.39,1.41,1.43,1.45,1.47$, $1.49,1.51,1.548,1.558,1.568,1.578$, and 1.588 . The other parameters are the same with those in Fig. 4.

In the ATR technique with a BK7-prism, the response of the multilayer configuration based on $\mathrm{Au}$ as an active metal and LHM-bulk and mediated with GaAs predicts an optical amplification that can be turned on the variation of RI. This amplification measured versus the incident angle is correlated to 
the SPR mode excited around the incident angle of $41.830^{\circ}$. The above effect was observed by the authors of [34] where the obtained optical amplification did not exceed $10^{3}$. The experimental use helps realize applications in precise processing and sensors. It was reported in [35] that the Goos-Hànchen shift was also sensitive to the change in refractive index and could be used as a different mechanism for sensing. In Figs. 4 and 5, the shift in the resonance angle for a variation $\Delta n_{s}=0.004$ remains constant in angular sensitivity that is in the order of $66^{\circ} / \mathrm{RIU}$.

\section{Conclusions}

The sensing properties evaluated from the reflectance curves on two arrangements between gold and LHM are investigated theoretically. Based on the simulated results, it has been shown that under the effect of RI of the sensing medium, gold as an active medium favors the presence of a high resolution required in biosensing. In appropriate conditions of the multilayer thicknesses, a single SPR of a giant intensity up to $10^{4}$ is sustained by the structure through the sensing medium of RI as 1.558. The later expected due to the Goos-Hànchen effect confirms the functionality of the structure to operate as an optical amplifier and an active tunable filter.

Open Access This article is distributed under the terms of the Creative Commons Attribution 4.0 International License (http://creativecommons.org/ licenses/by/4.0/), which permits unrestricted use, distribution, and reproduction in any medium, provided you give appropriate credit to the original author(s) and the source, provide a link to the Creative Commons license, and indicate if changes were made.

\section{References}

[1] X. Y. Dai, L. Y. Jiang, and Y. J. Xiang, "Tunable THz angular/frequency filters in the modified Kretschmann-Raether configuration with the insertion of single layer graphene," IEEE Photonics Journal, 2015, 7(2): 1-8.

[2] Y. A. Akimov and W. S. Koh, "Resonant and nonresonant plasmonic nanoparticle enhancement for thin-film silicon solar cells," Nanotechnology, 2010,
21(23): 235201.

[3] Y. A. Akimov, K. Ostrikov, and E. P. Li, "Surface plasmon enhancement of optical absorption in thin-film silicon solar cells," Plasmonics, 2009, 4(2): 107-113.

[4] F. F. Ren, K. W. Ang, J. F. Song, Q. Fang, M. B. Yu, G. Q. Lo, et al., "Surface plasmon enhanced responsivity in a waveguided germanium metal-semiconductormetal photodetector," Applied Physics Letters, 2010, 97(9): 091102-1-091102-3.

[5] L. Tang, S. E. Kocabas, S. Latif, A. K. Okyay, D. S. Ly-Gagnon, K. C. Saraswat, et al., "Nanometre-scale germanium photodetector enhanced by a near-infrared dipole antenna," Nature Photonics, 2008, 2(4): 226-229.

[6] B. Rothenhausler and W. Knoll, "Surface plasmon microscopy," Nature, 1988, 332(6165): 615-617.

[7] R. C. Jorgenson and S. S. Yee, "A fiber-optic chemical sensor based on surface plasmon resonance," Sensors \& Actuators B-Chemical, 1993, 12(3): 213-220.

[8] C. Nylander, L. Bo, and T. Lind, "Gas detection by means of surface plasmons resonance," Sensors \& Actuators, 1982, 3(82): 79-88.

[9] L. Bo, C. Nylander, and I. Lunstrom, "Surface plasmon resonance for gas detection and biosensing," Sensors \& Actuators, 1983, 4(83): 299-304.

[10] E. Kretschmann and H. Raether, "Radiative decay of non-radiative surface plasmons excited by light," Zeitschrift Für Naturforschung A, 1968, 23(12): 2135-2136.

[11] J. Homola, S. S. Yee, and G. Gauglitz, "Surface plasmon resonance sensors: review," Sensors \& Actuators B-Chemical, 1999, 54(1-2): 3-15.

[12] J. Homolar, Surface plasmon resonance based sensors series on chemical sensors and biosensors. Berlin, Germany: Springer-Verlag, 2006: 1-251.

[13] X. D. Hoa, A. G. Kirk, and M. Tabrizian, "Towards integrated and sensitive surface plasmon resonance biosensors: a review of recent progress," Biosensors \& Bioelectronics, 2007, 23(2): 151-160.

[14] P. K. Maharana and R. Jha, "Chalcogenide prism and graphene multilayer based surface plasmon resonance affinity biosensor for high performance," Sensors \& Actuators B-Chemical, 2012, 169(13): 161-166.

[15] H. S. Leong, J. Guo, R. G. Lindquisu, and Q. H. Liu, "Surface plasmon resonance in nanostructured metal films under the Kretschmann configuration," Journal of Applied Physics, 2009, 106(12): 124314-1-124314-5.

[16] V. G. Veselago, "The electrodynamics of substances with simultaneously negative values of $\varepsilon$ and $\mu$," Soviet Physics Uspekhi, 1968, 10(4): 509-514. 
[17] J. B. Pendry, A. J. Holden, D. J. Robbins, and W. J. Stewart, "Magnetic from conductors and enhanced nonlinear phenomena," IEEE Transactions on Microwave Theory Techniques, 1999, 47(11): 2075-2084.

[18] M. Schueler, C. Mandel, M. Puentes, and R. Jakoby, "Metamaterial inspired microwave sensors," IEEE Microwave Magazine, 2012, 13(2): 57-68.

[19] T. Chen, S. Y. Li, and H. Sun, "Metamaterials application in sensing," Sensors, 2012, 12(3): 2742-2765.

[20] J. J. Yang, M. Huang, H. Tang, J. Zeng, and L. Dong, "Metamaterial Sensors," International Journal of Antennas and Propagation, 2013, 2013(4): 1-16.

[21] A. Upadhyay, Y. K. Prajapati, V. Singh, and J. P. Saini, "Sensitivity estimation of metamaterial loaded planar waveguide sensor," Optical and Quantum Electronics, 2015, 47(7): 2277-2287.

[22] A. Upadhyay, Y. K. Prajapati, V. Singh, and J. P. Saini, "Comprehensive study of reverse index waveguide based sensor with metamaterial as a guiding layer," Optics Communications, 2015, 348: 71-76.

[23] N. I. Zheludev, "The road ahead for metamaterials," Science, 2010, 328(5978): 582-583.

[24] S. Pal, Y. K. Prajapati, J. P. Saini, and V. Singh, "Resolution enhancement of optical surface plasmon resonance sensor using metamaterial," Photonic Sensors, 2015, 5(4): 330-338.

[25] S. Pal, Y. K. Prajapati, J. P. Saini, and V. Singh, "Sensitivity enhancement of metamaterial-based SPR biosensor for NIR," Optica Applicata, 2016, 46(1): 131-143.

[26] Y. K. Prajapati, A. Yadav, A. Verma, V. Singh, and J. P. Saini, "Effect of metamaterial layer on optical surface plasmon resonance sensor," OptikInternational Journal for Light and Electron Optics, 2013, 124(18): 3607-3610.
[27] M. Yamamoto, "Surface plasmon resonance (SPR) theory: tutorial," Encyclopedic Reference of Immunotoxicology, 2010, 14(4): 388-398.

[28] J. Guo, P. D. Keathley, and J. T. Hastings, "Dual-mode surface-plasmon-resonance sensors using angular interrogation," Optics Letters, 2008, 33(5): 512-514.

[29] S. Szunerist, X. Castel, and R. Boukherroub, "Surface plasmon resonance investigation of silver and gold films coated with thin indium tin oxide layers: Influence on stability and sensitivity," Journal of Physical Chemistry C, 2008, 112(40): 15813-15817.

[30] A. D. Rakic, A. B. Djurisic, J. M. Elazar, and M. L. Majewski, "Optical properties of metallic films for vertical-cavity optoelectronic devices," Applied Optics, 1998, 37(22): 5271-5283.

[31] S. M. Xiao, V. P. Drachev, A. V. Kildishev, X. J. Ni, U. K. Chettiar, H. K. Yuan, et al., "Loss-free and active optical negative-index metamaterials," Nature, 2010, 466(7307): 735-738.

[32] K. M. Byun, S. J. Kim, and D. Kim, "Grating-coupled transmission-type surface plasmon resonance sensors based on dielectric and metallic gratings," Applied Optics, 2007, 46(23): 5703-5708.

[33] G. Gupta and J. Kondon, "Tuning and sensitivity enhancement of surface plasmon resonance sensor," Sensors \& Actuators B-Chemical, 2007, 122(2): 381-388.

[34] N. Goswami, A. Saha, and A. Ghosh, "Optical amplification with surface plasmon resonance and total internal reflection in gold nanostructure with BK7 parallel slab," International Journal of Chemtech Research, 2014, 7(3): 1148-1153.

[35] X. Yin, L. Hesselink, H. Chin, and D. A. B. Miller "Temporal and spectral nonspecularities in reflection at surface plasmon resonance," Applied Physics Letters, 2006, 89(4): 041102-1-041102-3. 